\title{
Synthesis and Characterization of Polycarbonate-containing All-organic High- $\chi$ Block Copolymers for Directed Self-assembly
}

\author{
Ankit Vora,* Anindarupa Chunder, Melia Tjio, Teddie Magbitang, Elizabeth Lofano, \\ Noel Arellano, Kristin Schmidt, Khanh Nguyen, Joy Cheng, and Daniel P. Sanders
}

\author{
IBM Research - Almaden, 650 Harry Road, San Jose, CA 95120 \\ avora@us.ibm.com
}

\begin{abstract}
To extend the scaling beyond the most widely used block copolymer (BCP), poly(styrene)-block-poly(methyl methacrylate) (PS-b-PMMA), a new organic high- $\chi$ BCP based was developed. Polystyrene-b-polytrimethylene carbonate (PS-b-PTMC) BCP was synthesized using ring opening polymerization (ROP) of trimethylene carbonate from hydroxy-functional polystyrene (PS-OH) with diazabicyclo[5.4.0]undec-7-ene (DBU) as the base catalyst. The resulting BCP was characterized by ${ }^{1} \mathrm{H}$ nuclear magnetic resonance (NMR) and gel permeation chromatography (GPC) to confirm the complete chain extension of the polystyrene macroinitiator. For the DBU catalyzed BCP, the GPC trace revealed a trimodal distribution indicating the presence of coupling product and homo-PTMC impurity. Using purification techniques, homo-PTMC impurity was isolated to afford purified BCP. Thin-film morphologies of the non-purified and the purified BCPs on poly(methyl methacrylate) (PMMA) coated substrates followed by short thermal annealing were characterized using atomic force microscopy (AFM) analysis. The non-purified BCP showed island morphology with a step-height of $17 \mathrm{~nm}$ whereas the purified PS-b-PTMC showed a flat film with parallel cylinders of $16.4 \mathrm{~nm}$ pitch row-to-row distance. The ability for the PS-b-PTMC to self-assemble below 20-nm pitch resolution confirms the high interaction parameter, $\chi$, between the PS and PTMC blocks and is a promising candidate as a high- $\chi$ BCP for directed self-assembly (DSA) application.
\end{abstract}

Keywords: Ring opening polymerization, organic high- $\chi$ block copolymers, polystyrene-b-polytrimethylene carbonate, directed self-assembly

\section{Introduction}

Directed self-assembly (DSA) is one of the most promising candidates for resolution enhancement for advanced lithography node [1]. While PS-b-PMMA is the most widely used block copolymer for DSA, the minimum half-pitch of this BCP is limited to $\sim 10 \mathrm{~nm}$ because of the low interaction parameter $\chi$ between PS and PMMA blocks. In order to obtain block copolymers with a smaller natural period, Lo, many new BCP platforms with higher $\chi$ parameters between their blocks have been investigated [2]. Several high- $\chi$
BCPs like PS- $b$-PDMS [3], PS-b-PTMSS [4], and PLA- $b$-PTMSS [5] having at least one inorganic block have been developed to increase the dry etch selectivity of the self-assembled nanostructures. On the other hand, organic-organic high- $\chi$ BCPs like PS- $b$-PEO [6], PS- $b$-P2VP [7], PaMS- $b$-PHOST [8], PS- $b$-PLA [9], partially epoxidized PS- $b$-PIE [10], poly(ethyleneoxide$b$-fluorinated methacrylate) [11] and PS- $b$-PHEMA [12] have also been developed to enable sub-10 nm half-pitch resolution for DSA.

Herein, we report a new class of block 
copolymers based on aliphatic polycarbonates for directed self-assembly. In this work the synthesis, purification and characterization of an all-organic, polystyrene-b-polytrimethylene carbonate (PSb-PTMC) high- $\chi$ BCP platform for DSA with sub-10 nm half-pitch resolution is reported. The impact of BCP purification on thin-film uniformity of the self-assembled features will be discussed.

\subsection{Materials, Instruments and} Characterization

Trimethylene carbonate (TMC) was received from Richman Chemical, Lower Gwynedd, PA 19002. The monomer was crystallized three times from toluene followed by drying under vacuum for two days to remove any residual toluene. The monomer was stored in a desiccator when it was not used for polymerization. Hydroxyl-end functional polystyrene (PS-OH, Mn $=6600$, PDI $=1.05)$ and AZEMBLYTM NLD-303 (PMMA brush solution) was a obtained as a gift from EMD Performance Materials Corp. Diazabicyclo[5.4.0] undec-7-ene (DBU) was obtained from Sigma-Aldrich and was distilled over calcium hydride under vacuum prior to using. Anhydrous dichloromethane (DCM) was received from Sigma-Aldrich and was used as received. Gel permeation chromatography was performed using THF as an eluent and calibrated with polystyrene standards. Morphology of the BCP thin films was characterized by atomic force microscopy (AFM) on Digital Instruments 3100 under ambient conditions. Pitch of block copolymer features were derived from FFT image processing of AFM images.

\subsection{Synthesis of polystyrene-} b-polytrimethylene carbonate (PS-b-PTMC) block copolymer.

Hydroxy-terminated polystyrene macroinitiator (PS-OH, $0.15 \mathrm{~g}, 0.028 \mathrm{mmol}, \mathrm{Mn}=6600$, PDI $=$ 1.05), TMC (0.192 g, $1.88 \mathrm{mmol})$ and DCM (1.90 $\mathrm{ml}$ ) were added to an oven-dried $4 \mathrm{ml}$ glass vial equipped with a magnetic stir bar. The reaction mixture was stirred until the PS-OH macroinitiator and TMC were completely dissolved in DCM, upon which ring opening polymerization catalyst DBU (17.5 mg, $0.113 \mathrm{mmol})$ was added. The reaction mixture was stirred at room temperature for 17 hours in a $\mathrm{N}_{2}$ glove box. The reaction was stopped by adding DCM $(1 \mathrm{ml})$, TEA $(0.1 \mathrm{ml}$, $0.388 \mathrm{mmol})$ and acetyl chloride $(0.02 \mathrm{ml}, 0.28$ mmol). The reaction was further stirred for two hours at room temperature. The block copolymer was isolated by precipitating the reaction mixture in methanol. The product was collected in a frit funnel by removing methanol under vacuum. The resulting solids were redissolved in THF to form a $20 \mathrm{wt} \%$ solution and reprecipitated in methanol. The solid was collected in a frit funnel and dried under vacuum at $40^{\circ} \mathrm{C}$ for two hours to obtain the PS-b-PTMC block copolymer. Mn $($ GPC $)=14100$, PDI $=1.08$. The PS block had $\mathrm{Mn}=6600$ (by $\mathrm{NMR}$ ) and a degree of polymerization $(\mathrm{DP})=63$. The PTMC block had $\mathrm{Mn}=7600$ and $\mathrm{DP}=74$, indicating $\sim 90 \%$ conversion of TMC. The volume fraction of PTMC block, $V f_{\text {PTMC }}$ was $\sim 0.47\left(\delta_{\text {PTMC }}\right.$ $=1.30 \mathrm{~g} / \mathrm{cm}^{3}$ ).

\subsection{Purification of PS-b-PTMC BCP.}

The crude PS-b-PTMC obtained above was further purified to remove any homopolymer of TMC. The resulting BCP had Mn $(\mathrm{GPC})=14,400$, PDI $=1.05$. The PS block had $\mathrm{Mn}=6600$ and $\mathrm{a}$ degree of polymerization $(\mathrm{DP})=63$. The PTMC block had $\mathrm{Mn}=4600$ (by ${ }^{1} \mathrm{H}$ NMR) and $\mathrm{DP}=47$. The volume fraction of PTMC block, $\mathrm{Vf}_{\text {PTMC }}$ of the purified PS-b-PTMC was $\sim 0.36$.

\subsection{Fabrication of underlayer and BCP coated substrates}

A solution AZEMBLYTM NLD-303 (PMMA brush solution) was spin coated with $2000 \mathrm{rpm}$ on a silicon wafer. The coated wafer was baked at $250^{\circ} \mathrm{C}$ for 2 minutes prior to a solvent rinse with PGMEA to form the UL coated substrate. Film thickness of the baked underlayer films was measured as $5 \mathrm{~nm}$ with Nanospec Reflectometer. PS-b-PTMC BCPs before and after purification were dissolved in PGMEA at a concentration of $1.2 \mathrm{wt} \%$ based on total weight of the solution. The solution was filtered (0.2 micrometer PTFE filter) and spin coated at $2000 \mathrm{rpm}$ rate on the PMMA brush coated UL substrate. The BCP thin films were subsequently annealed at $170^{\circ} \mathrm{C}$ for 5 minutes and immediately cooled to room temperature and analyzed by AFM.

\section{Results and Discussion}

3.1. PS-b-PTMC Block Copolymer Synthesis

To enable sub-10 nm half-pitch resolution for future patterning applications, polycarbonate-based BCP platform was developed by ring opening polymerization (ROP) of trimethylene carbonate (TMC) from hydroxyl-functional polystyrene 
macroinitiator using DBU as the base catalyst [13]. The reaction was carried out in a $\mathrm{N}_{2}$ filled glove box at room temperature until $\sim 90 \%$ conversion of TMC monomer. The resulting polymer had $\mathrm{Mn}(\mathrm{GPC})=14,100$ and PDI $=1.08$. The block lengths of the BCP was determined by ${ }^{1} \mathrm{H}$ NMR and was calculated as PS(6600)-b-PTMC(7600). The volume fraction of PTMC block of the BCP, $\mathrm{Vf}_{\text {PTMC }}$, was $\sim 0.47\left(\delta_{\text {PTMC }}=1.30 \mathrm{~g} / \mathrm{cm} 3\right)$, indicating a lamellae-forming BCP. Figure 1 shows the GPC trace for the $\mathrm{PS}-\mathrm{OH}$ macroinitiator (red) and the resulting PS-b-PTMC (blue) BCP. As it can be seen from the GPC trace, the DBU catalyzed AROP of TMC resulted in trimodal distribution.

\section{Base (DBU) Catalyzed ROP of TMC}

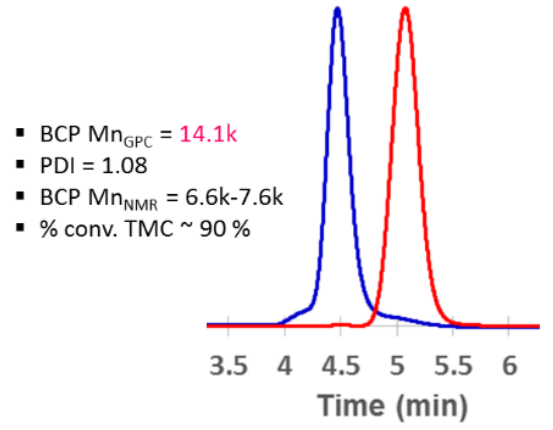

Figure 1. GPC traces for PS-OH (red), and PS-b-PTMC BCP (blue).

It has been previously reported that the ROP of aliphatic carbonates with highly active catalysts like 1,5,7-triazabicyclo[4.4.0]dec5 -ene (TBD) and DBU results in broadening of molecular weight distribution and bimodal GPC traces with high molecular weight shoulder due to transcarbonation of the polymer chains $[14,15]$. In our work on ring opening of TMC using PS-OH macroinitiator, in addition to the high molecular weight shoulder at high monomer conversion, a low molecular weight tail was also observed. We attribute this tail due to the formation of PTMC oligomers and homopolymers due to back-biting of the living polymer chains resulting from intramolecular transcarbonation [16].

\subsection{Purification of PS-b-PT MC BCP}

Access to highly pure BCPs is key for good DSA performance. DBU catalyzed BCP was purified to remove oligomers and homopolymer of TMC formed during the AROP. Figure 2 shows the GPC trace for the
PS-OH macroinitiator (red) and the purified PS-b-PTMC (blue) BCP. It was noticed that the low molecular weight tail of the BCP was eliminated after purficiation, resulting in a cleaner BCP with narrower PDI than the non-purified BCP. The block lengths of the purified BCP was determined by ${ }^{1} \mathrm{H} \quad \mathrm{NMR}$ as PS(6600)b-PTMC(4600) confirming the presence of homo-PTMC impurities in the BCP. The volume fraction of the PTMC block after BCP purification was $\sim 0.36$, indicating a cylinder-forming $\mathrm{BCP}$ with PS matrix and PTMC cylinders.

\section{Purified PS-b-PTMC}

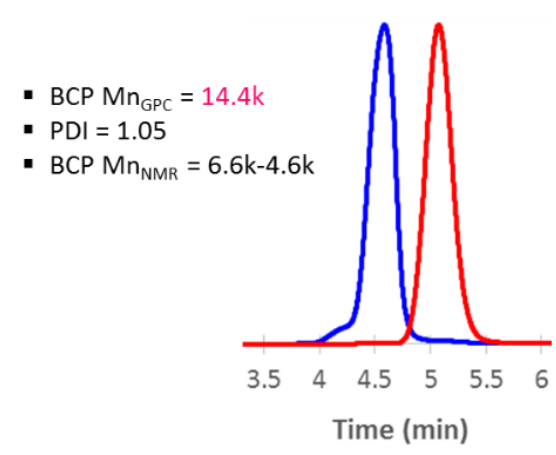

Figure 2. GPC traces for PS-OH (red), and purified PS-b-PTMC BCP (blue).

3.3. Impact of $\mathrm{BCP}$ purification on thin-film morphology of PS-b-PTMC

To evaluate the impact of impurity on thin-film self-assembly of PS-b-PTMC BCP, $1.2 \mathrm{wt} \%$ solutions of both the non-purified and the purified BCPs in PGMEA were spin-coated at 2000 RPM on PMMA modified $\mathrm{Si}$ wafer coupons. The resulting substrates were annealed at $170{ }^{\circ} \mathrm{C}$ for $5 \mathrm{~min}$ and immediately cooled to room temperature. The morphologies of the resulting thin-films were studied by AFM. Figure $3 \mathrm{a}$ is a $5 \mu \mathrm{m} \times 5 \mu \mathrm{m}$ scan size image of PS-b-PTMC before purification $\left(\mathrm{M} n_{\mathrm{NMR}} \quad\right.$ PS-b-PTMC $=$ 6.6k-b-7.6k, Vf $f_{\text {РTMC }} \sim 0.47$ ) showing $17 \mathrm{~nm}$ islands type morphology throughout the thin-film.

Interestingly, in the case of purified $\mathrm{BCP}$ $\left(\mathrm{M} n_{\mathrm{NMR}}\right.$ PS-b-PTMC $=6.6 \mathrm{k}-\mathrm{b}-4.6 \mathrm{k}, \mathrm{Vf}_{\mathrm{PTMC}} \sim$ 0.36 ), the AFM height image (Figure $3 b$ ) of the thin film annealed at $170{ }^{\circ} \mathrm{C}$ for 5 minutes showed a very flat surface with no island type features as seen for the non-purified BCP. In fact, $1 \mu \mathrm{m} \times 1 \mu \mathrm{m}$ scan image (Figure $3 \mathrm{c}$, inset) shows fingerprint pattern corresponding 
to parallel cylinders of PTMC with PS matrix.

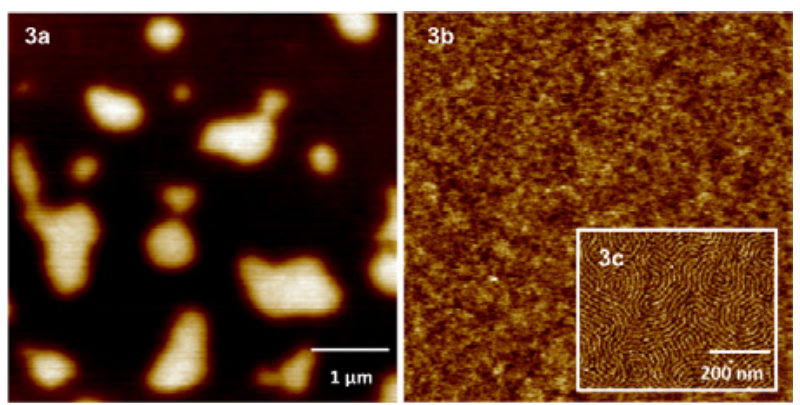

Figure 3. AFM height images of unpurified and purified PS-b-PTMC BCP on PMMA UL. PMMA is a preferential UL that wets the PS domain of the BCP. $3 \mathrm{a}$ shows islands of $17 \mathrm{~nm}$ pitch for the unpurified BCP $\left(\mathrm{Vf}_{\text {PTMC }} \sim 0.47\right) 3 \mathrm{~b}$ and $3 \mathrm{c}$ (inset) shows parallel cylinders of the purified $\mathrm{BCP}\left(\mathrm{Vf}_{\text {PTMC }} \sim 0.36\right)$ with 16.4 $\mathrm{nm}$ pitch row-to-row distance.

These results confirm that the PS-b-PTMC BCP synthesized by DBU catalyst has homo-PTMC impurities and using purification techniques, clean PS-b-PTMC BCP can be obtained. The thin film characterization results indicate that homo-PTMC impurity in the PS-b-PTMC BCP affects the morphology and highly clean BCPs are essential for DSA application.

\section{Conclusion}

Polycarbonate-containing high- $\chi$ BCP with sub-10 nm half-pitch were developed. DBU-catalyzed ring-opening polymerization from PS-OH macroinitiator resulted in impure block copolymers with significant amount of homo-PTMC. Using purification techniques, highly clean PS-b-PTMC BCP was obtained after eliminating the homopolymer impurity. Thin-film morphology studies of non-purified and purified BCPs revealed the importance of clean polymers for DSA application. While the non-purified BCP had a VfPTMC $\sim 0.47$ and showed $17 \mathrm{~nm}$ islands in AFM height image, the purified BCP with VfPTMC $\sim 0.36$ showed a very flat film with $16.4 \mathrm{~nm}$ pitch parallel cylinders.

Our ongoing work includes developing orientation control strategies for $\mathrm{BCP}$-substrate and BCP-air interfaces, grapho and chemoepitaxy DSA using the PS-b-Polycarbonate BCP platform and etch optimization to selectively remove the polycarbonate domain.

\section{Acknowledgement}

We extend our gratitude to Durairaj Baskaran and Guanyang Lin from EMD Performance Materials Corporation for providing PS-OH macroinitiator and AZEMBLYTM NLD-303 underlayer solution.

\section{References}

1. M. P. Stoykovich and P. F. Nealey, Mater.Today, 9 (2006) 20.

2. C. T. Black, R. Ruiz, G. Breyta, J. Y. Cheng, M. E. Colburn, K. W. Guarini, H.-C. Kim and Y. Zhang, IBM J. Res. Dev., 51 (2007) 605.

3. J. G. Son, K. W. Gotrik, C. A. Ross, ACS Macro Lett., 1 (2012) 1279.

4. C. M. Bates, T. Seshimo, M. J. Maher, W. J. Durand, J. D. Cushen, L. M. Dean, G. Blachut, C. J. Ellison, C. G. Willson, Science, 338 (2012) 775.

5. J. D. Cushen, C. M. Bates, E. L. Rausch, L. M. Dean, S. X. Zhou, C. G. Willson, C. J. Ellison, Macromolecules, 45 (2012) 8722.

6. Z. Q. Lin, D. H. Kim, X. D. Wu, L. Boosahda, D. Stone, L. LaRose, T. P. Russell, Adv. Mat., 14 (2002) 1373.

7. S. A. B. Shamsudin, G. Sakaguchi, M. Takenaka, H. Hasegawa, Macromol. Symp., 327 (2013) 72.

8. J. K. Bosworth, M. Y. Paik, R. Ruiz, E. L. Schwartz, J. Q. Huang, A. W. Ko, D. M. Smilgies, C. T. Black, C. K. Ober, ACS. Nano, 2 (2008) 1396.

9. I. Keen, A. Yu, H. H. Cheng, K. S. Jack, T. M. Nicholson, A. K. Whittaker, I. Blakey, Lamgmuir, 28 (2012) 15876.

10. S. Kim, P. F. Nealey, F. S. Bates, ACS. Macro. Lett., 1 (2012) 11.

11. H. Li, W. Gu, L. Li, Y. Zhang, T. P. Russell, E. B. Coughlin, Macromolecules, 46 (2013) 3737.

12. J. Cheng, R. A. Lawson, W. M. Yeh, N. D. Jarnagin, L. M. Tolbert, C. L. Henderson, Proc. SPIE, 8680 (2013) 86801W.

13. F. Nederberg, B.G.G. Lohmeijer, F. Liebfarth, R.C. Pratt, J. Choi, A.P. Dove, R.M. Waymouth, J.L. Hedrick, Biomacromolecules, 8 (2007), 153

14. S. Tempelaar, L. Mespouille, P. Dubois, A.P. Dove, Macromlecules,

15. R.C. Pratt, B.G.G. Lohmeijer, D.A. Long, R.M. Waymouth, J.L. Hedrick, JACS, 128 (2006), 4556.

16. H. Keul, "Handbook of Ring-opening Polymerization", Wiley-VCH, (2009), p308. 